\title{
Tecnura
}

http://revistas.udistrital.edu.co/ojs/index.php/Tecnura/issue/view/640

DOI: http://dx.doi.org/10.14483/udistrital.jour.tecnura.2014.SE1.a16

Estudio DE CASO

\section{Prototipo de emulación de movimiento humano mediante una plataforma bípeda humanoide}

\author{
Prototype for human movement emulation \\ by means of a bipedal humanoid platform
}

\author{
Milton Vicente Calderón Coy*, César Augusto Coronel Segrera** \\ Esperanza Camargo Casallas***
}

Citation / Para citar este artículo: Calderón Coy, M. V., Cesar Augusto, C. S., \& Camargo Casallas, E. (2014). Prototipo de emulación de movimiento humano mediante una plataforma bípeda humanoide. Revista Tecnura, Edición especial, 217-229.

Fecha de recepción: 16 de julio de 2013 / Fecha de aceptación: 7 de marzo de 2014

\section{Resumen}

En esta investigación se desarrolló una plataforma bípeda humanoide, cuya función es emular la marcha humana partiendo del principio de mantener el mínimo consumo energético minimizando el movimiento vertical de la pelvis. La plataforma se realizó utilizando herramientas de diseño y simulación como AutoCAD para la estructura mecánica, Solid-edge para el modelamiento tridimensional y Phyton para simular el movimiento de la plataforma. Estas herramientas suministraron las características físicas exactas de la plataforma, permitiendo generar el modelo cinemático. El modelo cinemático de la marcha se desarrolló utilizando MATLAB, así: a partir de una versión modificada de los parámetros de Denavit-Hartenberg se establece la cinemática directa, y la cinemática inversa se determina a partir de una red neuronal de tipo feedforward-backpropagation. Se obtuvo una plataforma bípeda humanoide con diez grados de libertad, capaz de realizar flexión-extensión en la cadera y rodilla, abducción-aducción en la cadera, flexión plantar-dorsal y pronación-supinación en el tobillo. La generación de trayectorias se obtiene en MATLAB mediante el ingreso del número de pasos, la longitud del paso, la altura del paso y una variable de tiempo que separará cada una de las secuencias o subpasos de la trayectoria. Como resultado se obtuvieron las trayectorias articulares en cadera, rodilla y tobillo en los tres planos, las cuales son acordes con la marcha humana y se evidenció con el robot bípedo humanoide.

Con esta investigación se espera establecer nuevos modelos para emular la marcha humana normal y patológica. Asimismo, esta es desarrollada por el grupo de investigación DIGITI de la Universidad Distrital Francisco José de Caldas.

Palabras clave: marcha humana, cinemática directa, cinemática inversa, Denavit-Hartenberg, robot bípedo.

\begin{abstract}
This research develops a bipedal humanoid platform, whose function is to emulate the human gait based on the principle of maintaining the minimum power consumption by minimizing the vertical movement
\end{abstract}

* Ingeniero en control electrónico. Investigador, grupo DIGITI de la Universidad Distrital Francisco José de Caldas. Bogotá, Colombia. Contacto: mvcalderonc@correo.udistrital.edu.co

** Ingeniero en control electrónico. Programador de hidrocarburos, Ecopetrol S.A.; investigador, grupo DIGITI de la Universidad Distrital Francisco José de Caldas. Bogotá, Colombia. Contacto: cesar.coronels@icloud.com

*** Ingeniera en control electrónico e instrumentación, especialista en instrumentación electrónica, estudiante de doctorado en ingeniería. Docente, Universidad Distrital Francisco José de Caldas. Bogotá, Colombia. Contacto: ecamargoc@udisrital.edu.co 
of the pelvis. The platform was developed using design and simulation tools such as AutoCAD for mechanical structure, Solid-edge for tridimensional modeling, and Phyton to simulate the movement of the platform. These tools provided accurate physical characteristics of the platform, allowing to generate the kinematic model. The gait kinematic model was developed using MATLAB, then; from a modified version of the Denavit-Hartenberg parameters the forward kinematics was established; and inverse kinematics is determined based on a feedforward backpropagation neuronal network. It was obtained a bipedal humanoid platform with ten degrees of freedom, capable of flexion-extension at the hip and knee, abduction-adduction at the hip,

\section{INTRODUCCIÓN}

Aunque existen distintos métodos para el análisis de la marcha como el cinético y cinemático, el análisis cinemático de la marcha es una herramienta fundamental que facilita el diagnóstico, tratamiento, seguimiento e implementación de métodos de rehabilitación. Este análisis se basa en la descripción y cuantificación de la variación en los desplazamientos de los segmentos del cuerpo y los centros de giro de las articulaciones. La recopilación de esta información permite obtener diferentes relaciones que se analizan en forma de reporte clínico y/o como herramienta de visualización virtual. En general, el proceso completo registra, procesa y cuantifica el movimiento del paciente en un contexto clínico.

El análisis cinemático estima las posturas durante la marcha, calculando la ubicación espacial del centro de masa del cuerpo humano y los centros de giro en cada una de las articulaciones que intervienen en el movimiento; esta estimación es suficiente para la mayor parte de las aplicaciones clínicas (Connor, Vaughan, Davis, \& Christopher, 1992), por lo que refiere un resultado cuantitativo y produce una representación virtual de la marcha, permitiendo al experto determinar alguna plantar-dorsal flexion and pronation-supination at the ankle. The trajectory generation is obtained in MATLAB by entering the number of steps, step length, step height and a time variable that will separate each of the sequences or sub-steps of the path. As a result, joint trajectories in hip, knee and ankle in three planes were obtained, which are in accordance with human gait, evidenced by means of the humanoid bipedal robot.

This research hopes to establish new models to emulate the normal and pathological human gait. Likewise, it is developed by research group DIGITI at District University Francisco José de Caldas.

Keywords: Human gait, forward kinematic, inverse kinematic, Denavit-Hartenberg, bipedal robot.

patología no detectable con técnicas convencionales, como la videogametría (Simon, 2004).

En medicina de rehabilitación es de gran importancia la estimación de la posición de los centros de giro de las articulaciones durante la marcha, ya que permite obtener la información clínica y un modelo observacional con un alto grado de veracidad (Aggarwal \& Cai, 1999; Wang, 2003).

En esta investigación se obtienen las trayectorias articulares durante las fases de marcha humana mediante cinemática directa obteniendo una representación virtual y se evidencia a partir de la construcción de un robot bípedo humanoide, de donde se obtienen las características físicas del modelo.

\section{BIOMECÁNICA DE LA MARCHA BÍPEDA}

Los métodos usados para estudiar robots, también pueden utilizarse para analizar la cinemática y dinámica humana. De esta forma, una de las suposiciones más comunes consiste en modelar el cuerpo humano como una cadena de eslabones rígidos, en donde cada segmento tiene diversas propiedades, como la longitud y la masa. Estos segmentos se unen mediante junturas que imitan las articulaciones humanas (cadera, rodilla y tobillo) 


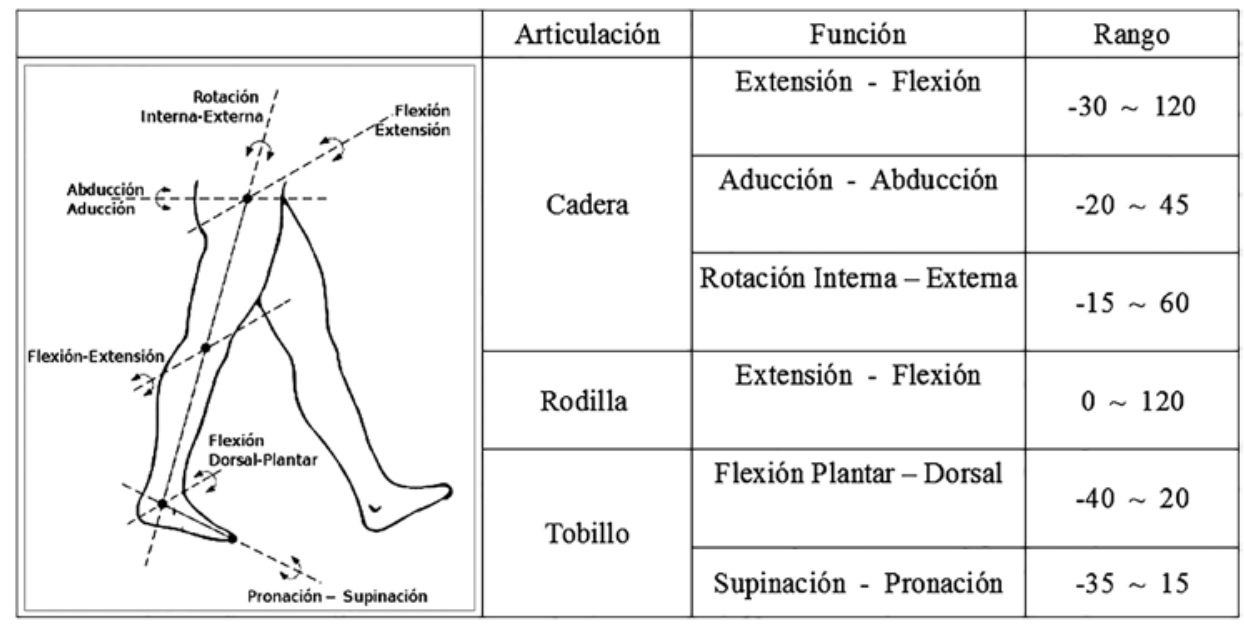

Figura 1. Ubicación de los grados de libertad y el rango de movilidad en cada articulación.

Fuente: Tomado de (Nordin, 2004).

considerando los seis grados de libertad distribuidos en las tres articulaciones y los rangos de movimiento básicos para el proceso de locomoción bípeda humanoide, partiendo de la biomecánica humana, como se muestra en la figura 1 (Nordin, 2004), (Luo et al., 2011).

\section{Locomoción humana}

La locomoción humana se constituye como un proceso cíclico; por tanto, repetitivo, por ello es dividida en fases (Kuo, 2007). La división de las fases de la locomoción depende del enfoque desde el cual se analiza el proceso de caminar, este puede ser:

\section{Análisis del cuerpo completo y como es soportado por cada pierna}

$\mathrm{Al}$ analizar el proceso de esta manera, se tiene que el ciclo del caminado consta de 2 fases: fase de soporte doble (doublesupportphase) y fase de soporte único (single supportphase) (Kuo, 2007; Jacquelin; Perry, 1992; Zanchi, 2000). El soporte único es realizado tanto por la pierna derecha como por la izquierda, lo que se identifica como soporte único derecho y soporte único izquierdo.
Fase de soporte único (Single Support Phase)

Durante esta fase, el pie al que se le hace referencia del soporte es el que se encuentra apoyado sobre el suelo y relativamente estacionario, como se observa en la figura 2; es el encargado de mantener el peso del cuerpo, y de ahí su nombre, "soporte". El comienzo de esta fase se puede distinguir cuando la otra pierna (la que no va a hacer el soporte) deja el suelo siendo los dedos del pie lo último en estar en contacto con el suelo, y termina cuando esa misma pierna vuelve a tocar el suelo por medio del talón (Kuo, 2007; Jacquelin; Perry, 1992; Zanchi, 2000).

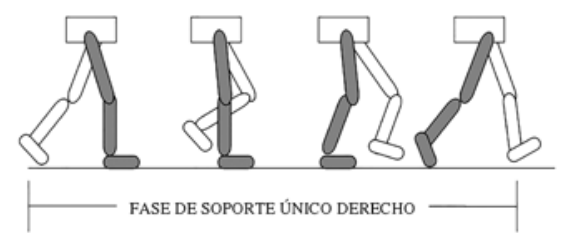

Figura 2. Fase de soporte único.

Fuente: Tomado de (Connor et al., 1992).

Fase de soporte doble (DoubleSupportPhase)

Se caracteriza porque los dos pies se encuentran en contacto con el suelo compartiendo el soporte 
del cuerpo. El comienzo de esta fase se reconoce cuando el talón de una pierna toca por primera vez el suelo y termina cuando los dedos del pie de la otra pierna se despegan del suelo. La duración de esta fase es bastante corta, pero indispensable ya que durante ella se hace el traspaso del soporte del cuerpo de una pierna a otra, tal como se puede ver en la figura 3 (Kuo, 2007; Jacquelin; Perry, 1992; Zanchi, 2000).

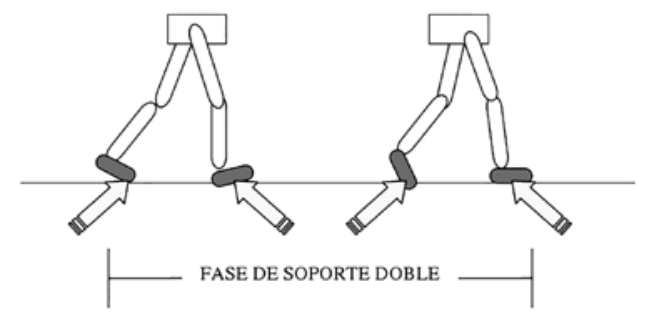

Figura 3. Fase de soporte doble.

Fuente: Tomado de (Connor et al., 1992).

Al analizar el movimiento que realiza cada pierna por separado se debe tener en cuenta que cada pierna es vista como un proceso independiente; por tanto, estaría dividido en fase de apoyo (stance phase) y fase de balanceo (swing phase). Cada una de estas fases se encuentra para las dos piernas, respectivamente (Kuo, 2007; Jacquelin; Perry, 1992; Zanchi, 2000).

\section{Fase de apoyo (Stance Phase)}

Esta fase representa el período de tiempo durante el cual la pierna analizada se encuentra en contacto con el suelo. Comienza cuando su talón toca el suelo y termina cuando los dedos del pie se despegan del suelo (Kuo, 2007; Jacquelin; Perry, 1992; Zanchi, 2000).

Fase de balanceo (Swing Phase)

Esta fase se distingue porque es el período de tiempo durante el cual la pierna analizada se encuentra en el aire balanceándose hacia el frente (Jacquelin Perry, 1992).
Luego de haber analizado el caminado humano y haber hecho una descripción general, es necesario abordar ciertos conceptos básicos sobre robótica ya que para lograr los objetivos propuestos se deben describir las piernas como una cadena articulada que implica un tratamiento de los temas fundamentales de esta área (Jacquelin Perry, 1992).

\section{METODOLOGÍA}

\section{Estructura mecánica de la plataforma}

La plataforma del robot bípedo se diseñó utilizando herramientas de diseño asistido por computador, como AutoCAD $®$ y Solid-Edge ${ }^{\circledR}$. Cada una de las piezas de la plataforma fue diseñada partiendo de la relación antropométrica del cuerpo humano, después se procedió al modelamiento en 3D de cada pieza; a modo de ejemplo se muestra la elaboración de una pieza en la figura 4.
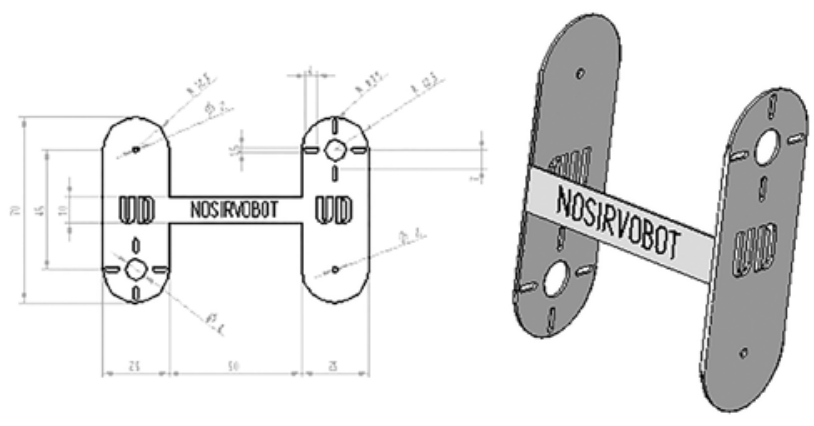

Figura 4. Modelamiento en 3D de una pieza de la plataforma.

Fuente: Elaboración propia.

Posteriormente se hizo el ensamble de todas las piezas del robot en Solid-Edge, tal como se observa en la figura 5. Una vez verificado el ensamblaje de las piezas en Solid-edge, se elaboraron cada una de estas conservando las medidas exactas de la simulación; en la figura 5 puede observarse la plataforma ensamblada totalmente. 


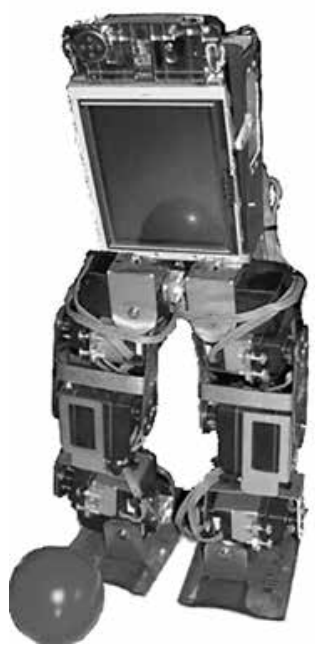

Figura 5. Modelo en 3D de la plataforma completa

Fuente: Elaboración del autor.

\section{Modelamiento de la plataforma}

\section{Extracción de las características físicas de la plataforma}

Las características físicas de los eslabones se extrajeron en forma indirecta con ayuda de Solid-Edge, lo cual se hizo mediante la ubicación de puntos de referencia en los ejes de los servomotores, tal como se puede observar a manera de ejemplo en la figura 6, en la ingle y en la base de los pies.

Una vez definidos estos puntos de referencia se efectuaron mediciones entre ellos para así
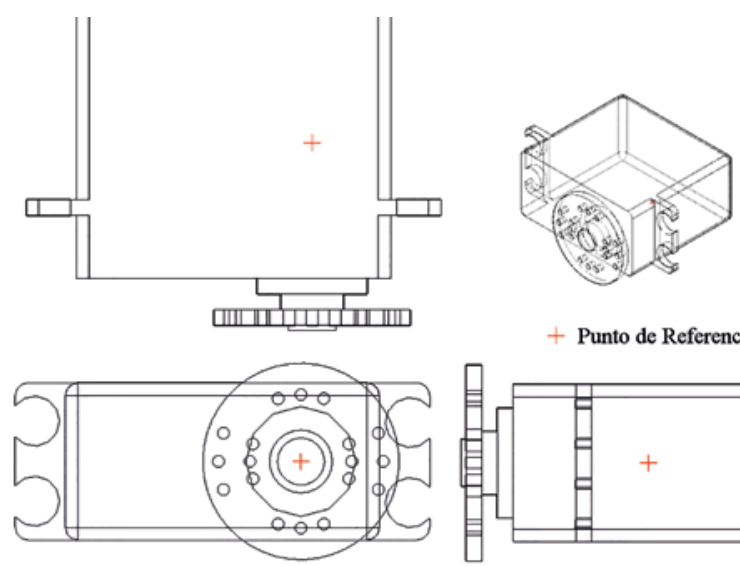

+ Punto de Referencia

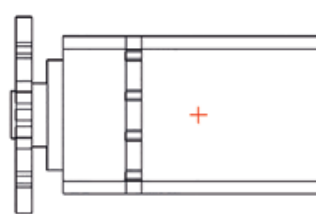

Figura 6. Ubicación del punto de referencia situado en el eje del servomotor.

Fuente: Elaboración del autor.

determinar las características de los eslabones; en la figura 7 se observa a manera de ejemplo el ensamble del tobillo y la cadera, en donde se toman los puntos de referencia antes mencionados y se traza una línea (Roja) que determinaría la geometría del eslabón en cada caso.

Así mismo, se hallaron los centros de volumen y masa de cada uno de los eslabones mediante la ayuda del mismo software, para lo cual fue necesario seleccionar el tipo de material utilizado en las piezas, en este caso se usó acero inoxidable y aluminio, los cuales presentan una densidad aproximada de $7.850 \mathrm{~kg} / \mathrm{m}^{3}$ y $2.700 \mathrm{~kg} / \mathrm{m}^{3}$, respectivamente.

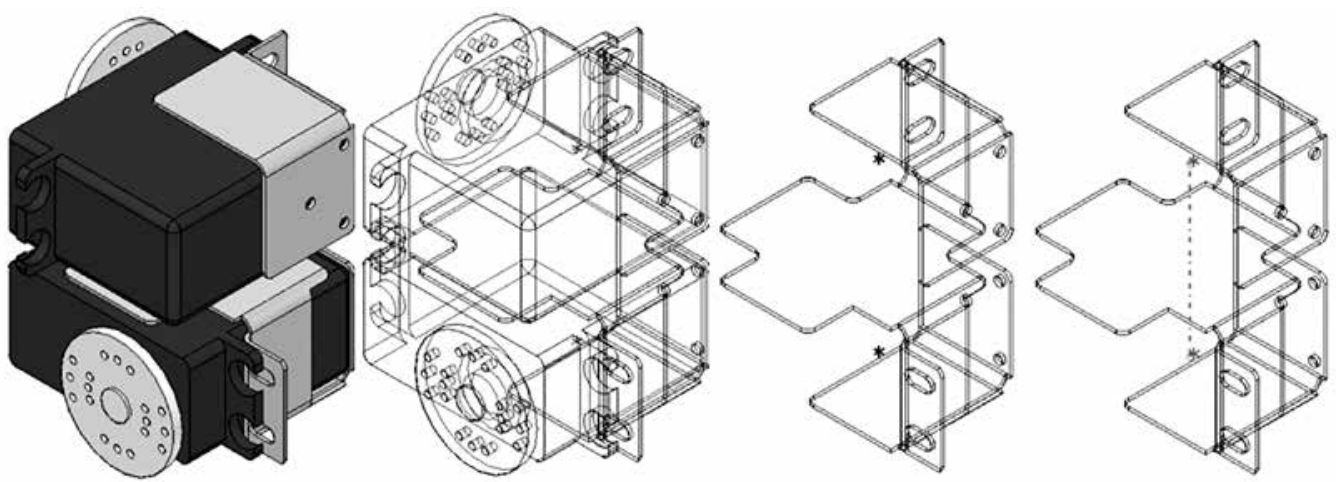

Figura 7. Ejemplo de caracterización de los eslabones.

Fuente: Elaboración del autor. 


\section{Modelo cinemático de la plataforma}

Una vez extraídas las características físicas de cada eslabón se procede a efectuar un modelo de cada uno de ellos y posteriormente su ensamble cinemático de la plataforma, a partir de los párametros de Denavit-Hartenverg.

\section{Denavit-Hartenberg}

El método de Denavit-Hartenberg consiste en un procedimiento sistemático mediante el cual se resuelve el problema de la cinemática directa de un manipulador constituido por un sistema de eslabones; el método se puede subdividir en tres fases, la primera consiste en la definición de los parámetros de D-H, luego se realiza la asignación de los sistemas de referencia y finalmente se obtienen las matrices de transformación homogénea (Gallego, Juan; Forner, 2008; V. Tikhanoff, P. Fitzpatrick, F. Nori, L. Natale, 2008).

Un eslabón siempre se encuentra relacionado o unido con otro eslabón a través de algún tipo de juntura. Por eso se puede considerar que la adecuada definición del eslabón y su juntura subsiguiente permiten la correcta descripción del sistema mecánico; en la figura 8 se observa un eslabón con la notación de Denavit-Hartenberg.

Para lograr esto, se han establecido 2 parámetros para describir el tamaño y la forma del eslabón, respectivamente, y 2 parámetros para describir la posición relativa de un eslabón con respecto a su predecesor. Estos cuatro parámetros se deben a Denavit y Hartenberg, por lo que se conocen como parámetros Denavit-Hartenberg.

Los parámetros que definen el tamaño y la forma del eslabón son: la longitud del eslabón a ${ }_{i-1}$ (link length) y la torción del eslabón $\mathrm{a}_{\mathrm{i}-1}$ (link twist). Mientras que los parámetros que describen la posición relativa del eslabón con respecto a su predecesor son: separación de eslabón $\mathrm{d}_{\mathrm{i}}$ (link offset) que expresa la distancia entre los dos eslabones, y el ángulo de juntura o articular $\mathrm{a}_{\mathrm{i}}$ (jointangle) que expresa el ángulo formado por los dos eslabones.

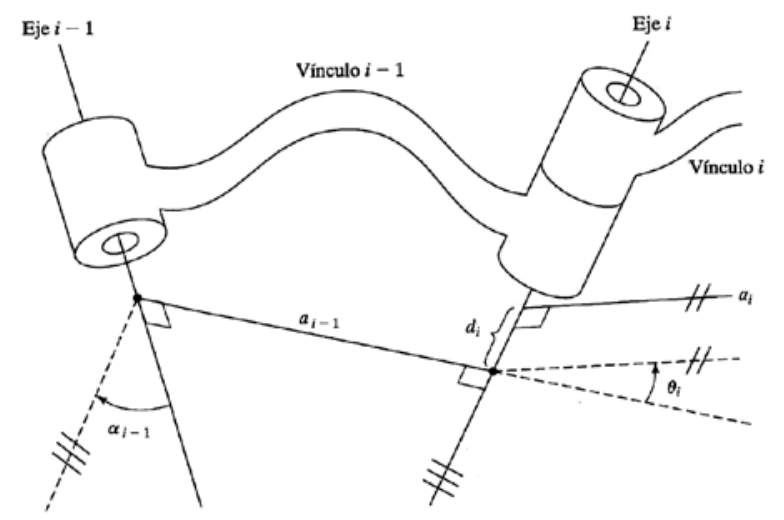

Figura 8. Parámetros de Denavit-Hartenberg.

Fuente: Tomado de (Sandin, 2003)

Una vez extraídos los parámetros de D-H del manipulador se elabora una tabla con los valores que describen cada eslabón, de la siguiente forma:

Tabla 1. Parametros de Denavit-Hartenberg

\begin{tabular}{lllll}
\hline$i$ & $\dot{a}_{i-1}$ & $a_{i-1}$ & $d_{i}$ & $\grave{e}_{i}$ \\
\hline 1 & & & & \\
\hline$\cdots$ & & & & \\
\hline$n$ & & & & \\
\hline
\end{tabular}

Y posteriormente se obtienen las matrices de transformación homogénea mediante la ecuación (1).

$$
{ }_{A}=\operatorname{rot}\left(X_{i}, \dot{a}_{i 1}\right) \operatorname{trans}\left(X_{i}, a_{i 1}\right) \operatorname{rot}\left(Z_{i}, \grave{e}_{i}\right) \operatorname{trans}\left(Z_{i}, d_{i}\right)
$$

Modificación de los parámetros de

Denavit-Hartenberg

El modelo matemático de los eslabones se realizó mediante una versión modificada de los parámetros de Denavit-Hartenberg (figura 9). La modificación efectuada se implementó ya que no se pudieron expresar los eslabones con la notación normal de cuatro parámetros. 


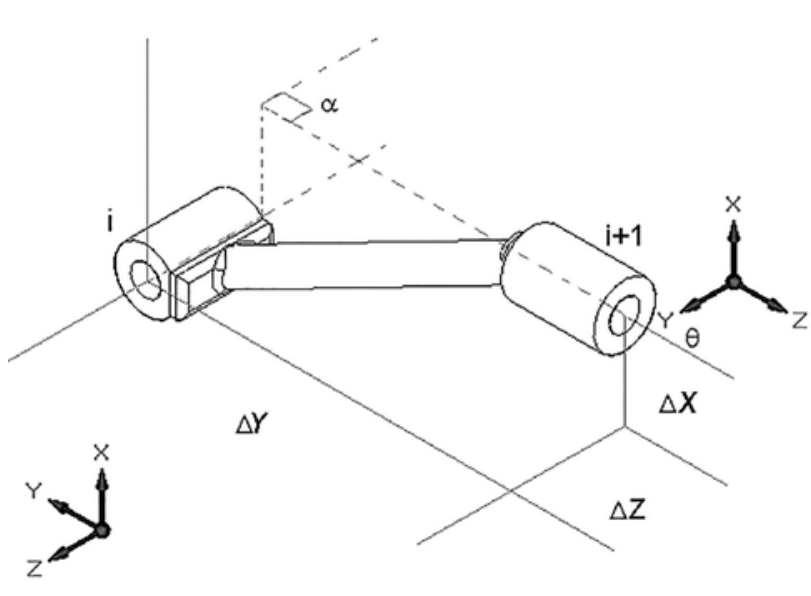

Figura 9. Modificación de los parámetros de Denavit-Hartenberg.

Fuente: Tomado de (Sandin, 2003)

Por lo que se tuvo en cuenta la rotación sobre el eje, tal como se muestra en la siguiente tabla 2.

Tabla 2. Conversión de parámetros según la rotación sobre el eje

\begin{tabular}{cccc}
\hline$\alpha$ & 0 & 90 & -90 \\
\hline $\mathrm{a}$ & $\Delta \mathrm{x}$ & $\Delta \mathrm{x}$ & $\Delta \mathrm{x}$ \\
\hline $\mathrm{d}$ & $\Delta \mathrm{y}$ & $-\Delta \mathrm{z}$ & $\Delta \mathrm{z}$ \\
\hline $\mathrm{p}$ & $\Delta \mathrm{z}$ & $\Delta \mathrm{y}$ & $-\Delta \mathrm{y}$ \\
\hline
\end{tabular}

Adicionando un parámetro mediante el cual se logró describir el eslabón.

$\alpha \rightarrow$ rotación entre y en el eje

$\mathrm{a} \rightarrow$ longitud del eslabón entre y en el eje

$p \rightarrow$ altitud del eslabón entre y en el eje

$\theta \rightarrow$ rotación entre y en el eje

d $\rightarrow$ profundidad del eslabón entre y en el eje

Obtención de los parámetros de

Denavit-Hartenberg

A partir de las modificaciones de los parámetros de Denavit-Hartenberg, se establecieron los parámetros de Denavit-Hartenber. (Ver las tablas 3 y 4).
Tabla 3. Parámetros de Denavit-Hartenberg modificada para la pierna izquierda.

\begin{tabular}{cccccc}
\hline $\mathrm{n}$ & $\alpha$ & $\mathrm{a}$ & $\mathrm{p}$ & $\theta$ & $\mathrm{d}$ \\
\hline 0 & $(-\pi) / 2$ & - Cmed & 0 & $\theta \_1+\pi / 2+\operatorname{corr}$ & 0 \\
\hline 1 & $\pi / 2$ & Tob & Tov & $\theta \_2$ & -Top \\
\hline 2 & 0 & $\mathrm{~h}$ & 0 & $\theta_{-} 3$ & hv \\
\hline 3 & 0 & Pn & - Pnp & $\theta \_4$ & -Pnv \\
\hline 4 & $\pi / 2$ & Tob & Top & $-\theta_{-} 5+\pi / 2+$ corr & Tov \\
\hline 5 & $(-\pi) / 2$ & 0 & 0 & 0 & -AltPie \\
\hline
\end{tabular}

Tabla 4. Parámetros de Denavit-Hartenberg modificada para la pierna derecha.

\begin{tabular}{cccccc}
\hline $\mathrm{n}$ & $\alpha$ & $\mathrm{a}$ & $\mathrm{p}$ & $\theta$ & $\mathrm{d}$ \\
\hline 0 & $(-\pi) / 2$ & Cmed & 0 & $-\theta \_1+\pi / 2$-corr & 0 \\
\hline 1 & $\pi / 2$ & Tob & Tov & $\theta \_2$ & Top \\
\hline 2 & 0 & $\mathrm{~h}$ & 0 & $-\theta \_3$ & $-\mathrm{hv}$ \\
\hline 3 & 0 & Pn & - Pnp & $\theta \_4$ & Pnv \\
\hline 4 & $\pi / 2$ & Tob & - Top & $\theta_{-} 5+\pi / 2$-corr & Tov \\
\hline 5 & $(-\pi) / 2$ & 0 & 0 & 0 & -AltPie \\
\hline
\end{tabular}

Las variables de construcción están relacionadas en la tabla 5.

Tabla 5. Variables de modelamiento.

\begin{tabular}{lcc}
\hline \multicolumn{1}{c}{ Sección } & Variable & Valor $[\mathbf{m m}]$ \\
\hline \multirow{2}{*}{ Cadera } & Cmed2 & 22.5 \\
\cline { 2 - 3 } Cadera & corr & \\
Tobillo & Tob & 22.7 \\
\cline { 2 - 3 } & Tov & 14.25 \\
\cline { 2 - 3 } Muslo & Top & 14.25 \\
\hline \multirow{2}{*}{ Pierna } & $h$ & 45 \\
\cline { 2 - 3 } & $h v$ & 3.5 \\
\hline Pie & $P n$ & 62.84 \\
\cline { 2 - 3 } & $P n p$ & 2.14 \\
\hline
\end{tabular}

Basándose en los parámetros obtenidos (tablas 3 y 4) se generaron las matrices de transformación homogénea que relacionan una articulación con la siguiente. 
Generación de las matrices de transformación homogénea

Las matrices de transformación homogénea se encuentran relacionadas a continuación y en cada una de ellas se encuentran las variables articulares que están agrupadas en las ecuaciones (2) y (3). La numeración de dichas variables se hizo de arriba a abajo usando MATLAB.

$$
\begin{gathered}
A I=\left[\begin{array}{l}
a i 1 \\
a i 2 \\
a i 3 \\
a i 4 \\
a i 5
\end{array}\right] \\
A D=\left[\begin{array}{l}
a d 1 \\
a d 2 \\
a d 3 \\
a d 4 \\
a d 5
\end{array}\right]
\end{gathered}
$$

Matriz de transformación del punto de referencia ubicado en el servomotor izquierdo uno referenciado al punto de referencia en la ingle (ecuación (4))

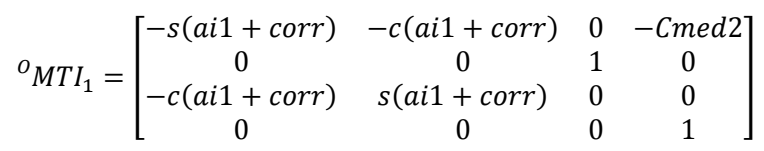

Matriz que relaciona el punto de referencia del servomotor dos con respecto al servomotor uno (ecuación (5)).

$$
{ }^{1} \mathrm{MTI}_{2}=\left[\begin{array}{cccc}
c(\text { ai2 }) & -s(a i 2) & 0 & T \\
0 & 0 & -1 & \text { Top } \\
s(a i 2) & c(a i 2) & 0 & \text { Tov } \\
0 & 0 & 0 & 1
\end{array}\right]
$$

Matriz de transformación que relaciona el servomotor tres al servomotor dos (ecuación (6)).

$$
{ }^{2} \mathrm{MTI}_{3}=\left[\begin{array}{cccc}
c(a i 3) & s(a i 3) & 0 & h \\
-s(a i 3) & c(a i 3) & 0 & 0 \\
0 & 0 & 1 & h v \\
0 & 0 & 0 & 1
\end{array}\right]
$$

Matriz que relaciona el servomotor cuatro al punto de referencia del servomotor tres de la pierna izquierda (ecuación (7)).

$$
{ }^{3} \mathrm{MTI}_{4}=\left[\begin{array}{cccc}
c(\text { ai4 }) & -s(\text { ai4 }) & 0 & P n \\
s(a i 4) & c(a i 4) & 0 & -P n p \\
0 & 0 & 1 & -P n v \\
0 & 0 & 0 & 1
\end{array}\right]
$$

Matriz que relaciona el servomotor cinco con respecto al punto de referencia situado en el servomotor cuatro (ecuación (8)).

$$
{ }^{4} M T I_{5}=\left[\begin{array}{cccc}
s(a i 5-c o r r) & -c(a i 5-c o r r) & 0 & T \\
0 & 0 & -1 & -T o v \\
c(a i 5-c o r r) & s(a i 5-c o r r) & 0 & T o p \\
0 & 0 & 0 & 1
\end{array}\right]
$$

Matriz de transformación que relaciona la base del pie con respecto al punto de referencia situado en el servomotor cinco (ecuación (9)).

$$
{ }^{5} M T I_{b p}=\left[\begin{array}{cccc}
1 & 0 & 0 & 0 \\
0 & 0 & 1 & - \text { AltPie } \\
0 & -1 & 0 & 0 \\
0 & 0 & 0 & 1
\end{array}\right]
$$

En las ecuación (10) a la (15), se muestran las matrices de transformación equivalentes para la pierna derecha.

$$
\begin{aligned}
& { }^{o}{ }_{M T D_{1}}=\left[\begin{array}{cccc}
s(a d 1+c o r r) & -c(a d 1+c o r r) & 0 & \text { cmed } 2 \\
0 & 0 & 1 & 0 \\
-c(a d 1+c o r r) & -s(a d 1+c o r r) & 0 & 0 \\
0 & 0 & 0 & 1
\end{array}\right] \\
& { }^{1} M T D_{2}=\left[\begin{array}{cccc}
c(\text { ad2 }) & -s(a d 2) & 0 & T \\
0 & 0 & -1 & -T o p \\
s(a d 2) & c(a d 2) & 0 & T o v \\
0 & 0 & 0 & 1
\end{array}\right] \\
& { }^{2} M T D_{3}=\left[\begin{array}{cccc}
c(a d 3) & s(a d 3) & 0 & h \\
-s(a d 3) & c(a d 3) & 0 & 0 \\
0 & 0 & 1 & -h v \\
0 & 0 & 0 & 1
\end{array}\right] \\
& { }^{3} \mathrm{MTD}_{4}=\left[\begin{array}{cccc}
c(\text { ad4 }) & -s(a d 4) & 0 & P n \\
s(a d 4) & c(a d 4) & 0 & -P n p \\
0 & 0 & 1 & P n v \\
0 & 0 & 0 & 1
\end{array}\right]
\end{aligned}
$$




$$
\begin{gathered}
{ }^{4} M T D_{5}=\left[\begin{array}{cccc}
-s(a d 5-c o r r) & -c(a d 5-c o r r) & 0 & T \\
0 & 0 & -1 & -T o v \\
c(a d 5-c o r r) & -s(a d 5-c o r r) & 0 & -T o p \\
0 & 0 & 0 & 1
\end{array}\right] \\
{ }^{5} M T D_{b p}=\left[\begin{array}{cccc}
1 & 0 & 0 & 0 \\
0 & 0 & 1 & - \text { AltPie } \\
0 & -1 & 0 & 0 \\
0 & 0 & 0 & 1
\end{array}\right]
\end{gathered}
$$

En la figura 10 se encuentra el orden en que se sitúan los marcos de referencia en la plataforma modelados en MATLAB.

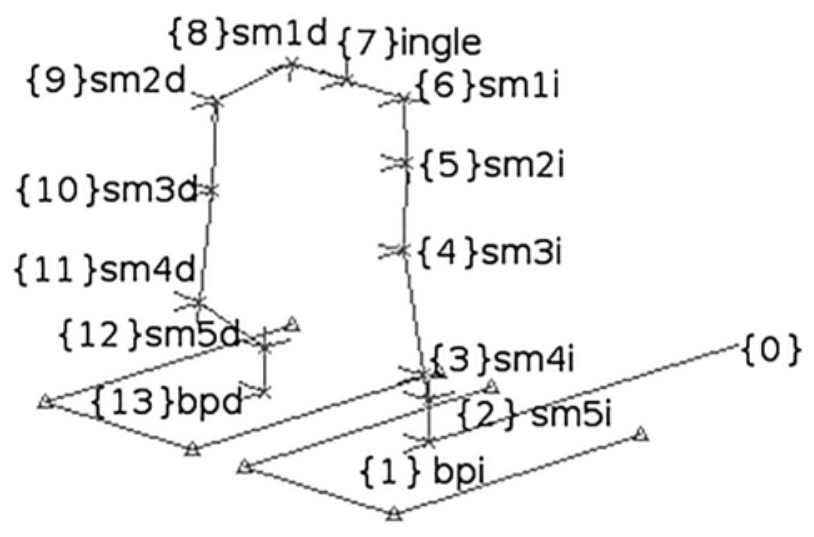

Figura 10. Ubicación de los puntos de referencia de la plataforma con respecto a un marco de referencia fijo.

Fuente: Elaboración propia.

Elaboración de la cinemática inversa mediante $R N$ Para simplificar el modelo de la plataforma se redujeron los grados de libertad, ya que se insertaron tres restricciones al modelo con lo cual el marco de referencia del pie de apoyo presenta el mismo marco de referencia que el origen.

La cinemática inversa se trabajó a través de redes neuronales, para ello se escogió la red neuronal de tipo feedforward-backpropagation, la cual tiene entre sus propiedades y aplicaciones ser utilizada en aproximación de funciones (Cravajal, 2003). La red neuronal utilizada tiene la topología que se aprecia en la figura 11.

La función de la red neuronal es devolver los valores de las variables articulares al ingresarles las posiciones deseadas para los pies y la ingle. Para lograr este objetivo se creó un juego de datos basado en la cinemática directa, en el cual se hallan dos subconjuntos de datos uno de los cuales contiene las posiciones y orientaciones de la ingle y las posiciones de los pies, y el otro contiene los valores de las variables articulares (Pina, 2007).

Para crear estos conjuntos se evaluaron las funciones características de una sola pierna, haciendo una variación de 10 grados por vez en cada servomotor; luego de barrido el rango del servo se continuaba con el siguiente hasta completar el barrido de los cinco servos. Con esto se generó un conjunto de 22750 datos de prueba con sus respectivos objetivos.

La red neuronal fue diseñada y probada con los datos cinemáticos de la pierna izquierda; sin embargo, debido a la simetría existente, la red puede utilizarse con la pierna derecha efectuando dos cambios de signo.

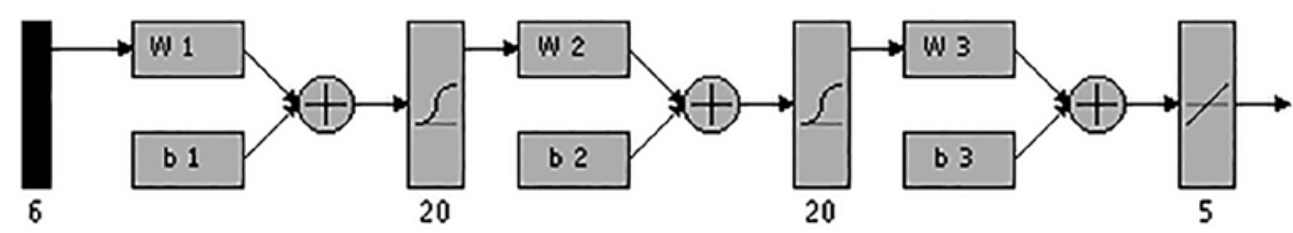

Figura 11. Topología de la red neuronal utilizada.

Fuente: Tomado de (Cravajal, 2003). 


\section{Construcción del robot virtual}

\section{Simulación en Python}

A partir de las características físicas de la plataforma y los subconjuntos de datos requeridos para mover cada uno de los servomotores se desarrolló un entorno de simulación en Phyton con el fin de cubrir expectativas de movimiento en cuanto a simulación en ambiente real; para esto el paso inicial fue diseñar WordSpace 3D usando la librería vPython de Python para entornos 3D (Pina, 2007).

Luego de tener desarrollado el entorno se procedió a elaborar y caracterizar una librería enfocada a las propiedades del servomotor. Estas propiedades correspondían al servomotor en su ambiente real, como peso, torque y velocidad.

Servomotor: Para el diseño del servomotor se hizo una caja dándole las propiedades físicas como peso y densidad, esto se logra a través de las librerías PyODE. El servomotor está creado con base en una simple caja en $3 \mathrm{D}$ con las respectivas dimensiones del servo usado; a este se le agregó una circunferencia en uno de los costados simulando el eje rotativo y dándole las cualidades de rotación al mismo.
Creadas las librerías de los servomotores y de las uniones se procedió a ensamblar el robot virtual posicionando cada uno de los elementos en sus respectivas coordenadas y haciendo las uniones en los puntos específicos, con la finalidad de generar todo como un conjunto. Con cada una de las piezas y el servomotor se inicia la construcción del robot.

Otra pieza fundamental es el tronco en donde va situada la PDA, es decir, el controlador electrónico del robot, la cual está compuesta de un objeto caja con 2 articulaciones en su parte inferior. Debido a esto es crucial la medición de peso del conjunto como tal para que la simulación sea lo más real posible.

El ensamble de la plataforma se hizo de abajo hacia arriba para no tener inconvenientes con las propiedades de la librería PyODE. La construcción completa se puede observar en la figura 12 .

En el entorno de simulación se diseñó un aplicativo llamado Servomanager, este se utilizó para la programación de los movimientos virtuales del robot. El Servomanager consiste en una interfaz que sea capaz de traducir el lenguaje escrito previamente establecido en movimientos representados en el modelo virtual.

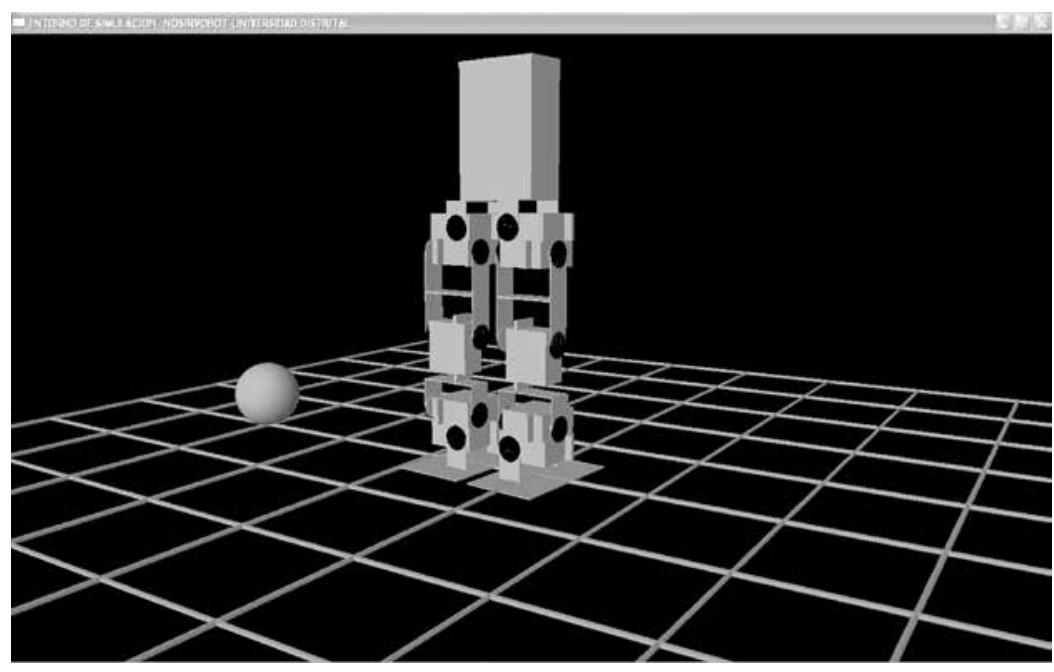

Figura 12. Modelo de la plataforma completa en el entorno de simulación.

Fuente: Elaboración del autor. 


\section{RESULTADOS}

\section{Sistema electrónico del robot}

El sistema electrónico está compuesto por una tarjeta controladora de servos, los servomotores, un acelerómetro y un computador o un asistente personal (PDA, por sus siglas en inglés); los servomotores son la fuente motriz de la plataforma ya que son los encargados de hacer las variaciones sobre las variables articulares con las cuales se logrará el desplazamiento de la plataforma; el acelerómetro es el encargado de medir la inclinación de la plataforma; la tarjeta controladora de servos es la encargada de emitir las señales de control a cada uno de los servomotores presentes en la plataforma, además es la encargada de hacer la lectura del acelerómetro y establecer comunicación con el computador o con la PDA y de este modo recibir las instrucciones que debe emitir a los servomotores con el fin de reproducir el movimiento deseado, y en algunos casos la función de enviar al computador la lectura del acelerómetro; el diagrama de bloques implementado se muestra en la figura 13.

La comunicación entre la tarjeta controladora de los servomotores y la PDA se hizo mediante una adaptación de voltajes entre las etapas de comunicación ya que la PDA funciona con 3.3 voltios como uno lógico, y la tarjeta controladora maneja voltajes TTL de 5 voltios.

Para efectos de la comunicación con la tarjeta controladora el puerto serial debió configurarse con la opción RST y CTS interconectados.

Fue necesario que los voltajes de lógica y los voltajes de alimentación de los servomotores provinieran de fuentes diferentes, ya que el ruido proveniente de los servomotores afecta la comunicación entre la tarjeta controladora de servos y la PDA.

\section{Control de estabilidad}

El modelo de control de la plataforma está compuesto por un sensor de estabilidad (el acelerómetro) y los actuadores (los servomotores), (Eiji, 2007; Sandin, 2003; Zivanovic, 2006).

El acelerómetro es uno de los transductores más versátiles, siendo el más común el piezoeléctrico por compresión. Este se basa en que cuando se comprime un retículo cristalino piezoeléctrico, se produce una carga eléctrica proporcional a la fuerza aplicada. En este caso se utilizó el acelerómetro LIS3L02AL, el cual posee medición en los 3 ejes, el sensor envía 3 señales correspondientes a cada

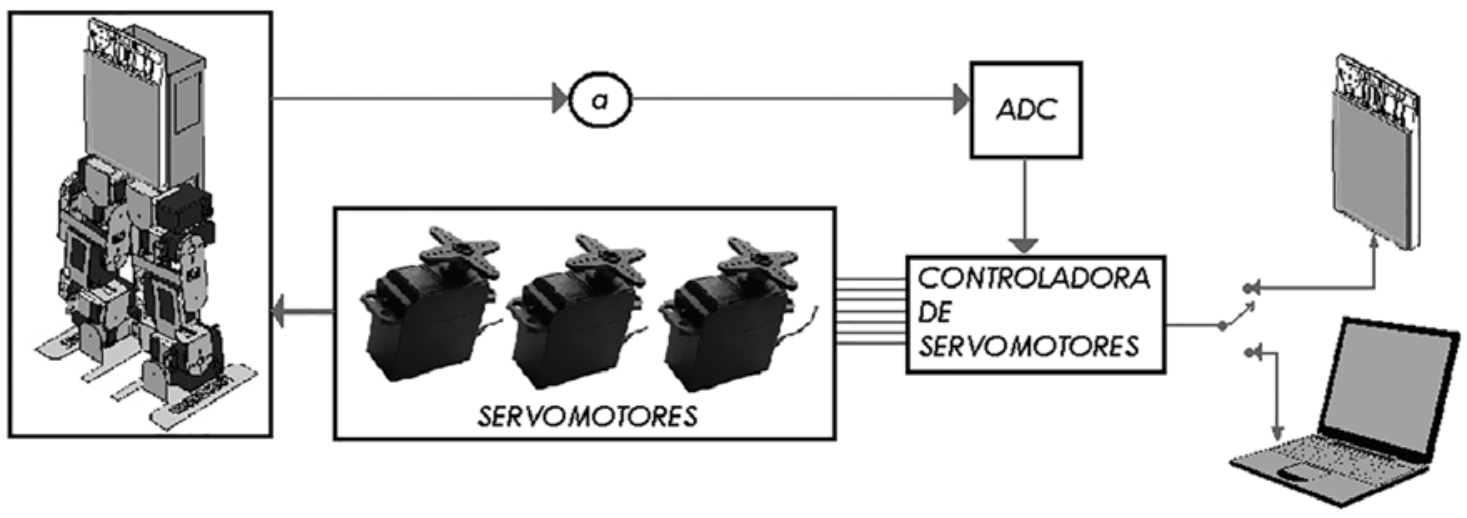

Figura 13. Modelo de control de la plataforma.

Fuente: Elaboración propia. 
uno de los ejes; estas señales varían según la orientación del robot. En cuanto a las señales de los acelerómetros configurados en posición de gravedad, se debe aclarar que cada vez que alguno de los ejes apunte en contra de la gravedad este tomará un valor positivo, y cuando apunte a favor de la gravedad este tomará un valor negativo y cuando se encuentre en el plano perpendicular a la gravedad, tendrá un valor neutro.

\section{Generación de trayectorias}

La generación de trayectorias se obtiene en MatLab mediante el ingreso de los cuatro parámetros antes mencionados: número de pasos, longitud, altura del paso y una variable de tiempo que separara cada una de las secuencias o subpasos de la trayectoria. Las trayectorias resultantes se muestran en la figura 14 .

En la figura 14 se pueden observar las trayectorias que seguirán los puntos de referencia ubicados en la ingle (morado), pie izquierdo (azul) y pie derecho (rojo), (a) esta la trayectoria tridimensional, en las figuras (b), (c) y (d) aparecen las trayectorias desde una vista superior, lateral y frontal, respectivamente.

Las trayectorias obtenidas se encuentran acordes con las trayectorias de la marcha humana.

\section{CONCLUSIONES}

Mediante la utilización de herramientas computarizadas se diseñó e implementó una plataforma bípeda humanoide, a la cual se le aplicó un análisis cinemático y con base en este se generaron componentes de software en Matlab, Python y Visual Basic Embeded, mediante los cuales se emuló el movimiento bípedo humanoide en la plataforma.

Se diseñó e implementó una plataforma de emulación del movimiento humano, con herramientas de diseño asistido por computador como lo es Solid-edge para el modelamiento tridimensional de la plataforma y para su construcción se utilizó AutoCAD en el proceso de diseño y corte de cada una de las piezas necesarias para ensamblar la plataforma.

El modelo cinemático de la plataforma se determinó mediante las características obtenidas con el uso de las herramientas mencionadas, con las cuales se obtienen los modelos de cada uno de los elementos que componen la plataforma como eslabones, motores, baterías, etc., y posteriormente se genera el modelo cinemático de la plataforma.

Los algoritmos para los movimientos básicos de las articulaciones se generaron mediante la

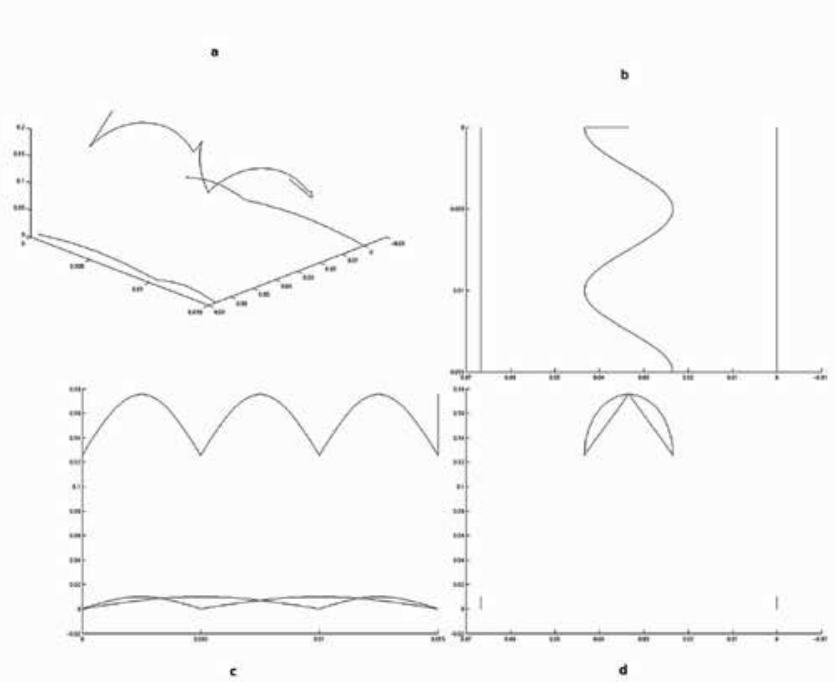

Figura 14. Trayectorias generadas para la plataforma.

Fuente: Elaboración del autor. 
comparación entre la señal de control y el ángulo en cada una de las articulaciones.

La generación de algoritmos complejos está a cargo de una función que genera las trayectorias cartesianas a cada uno de los puntos de referencia situados a lo largo de la plataforma, los cuales se ingresan a una red neuronal que genera las trayectorias articulares de cada una de las articulaciones de la plataforma; acto seguido se hace la conversión de las variables articulares a señales de control que serán enviadas a cada uno de los motores de la plataforma para así reproducir el movimiento programado.

Se integró a la plataforma un acelerómetro que sensa mediante sus propiedades intrínsecas de medición en los tres ejes, estas se envían a un lazo de control en el cual se toman las decisiones para la estabilidad del sistema.

En el momento del ensamblaje final de la plataforma debe tenerse en cuenta que los servomotores se encuentren en su posición media con los respectivos offsets calibrados, para la obtención de una perfecta simetría en los movimientos respecto a la pierna izquierda y la derecha.

Todos los movimientos deben ser previamente calculados en el lenguaje phyton para evitar choques mecánicos entre las piezas y del mismo modo desgastes de los servomotores innecesariamente.

\section{FINANCIAMIENTO}

Universidad Distrital Francisco José de Caldas.

\section{REFERENCIAS}

Aggarwal, J. K., \&Cai, Q. (1999). Human motion analysis: a review. Computer Vision and Image Understanding, 73(3), 428-440.

Connor, J. C. O.; Vaughan, C. L.; Davis, B. L., \& Christopher, L. (1992). DYNAMICS OF HUMAN GAIT (C. Vaughan, ed.) Africa (2nd ed., p. 153). Cape Town, South Africa: Kiboho Publisher. Retrieved from http://www.kiboho.co.za/GaitCD
Carvajal, J. (2003). Robótica. Aproximación al diseño (p. 37). Pamplona: Universidad de Pamplona.

Eiji, A. T. A. (ed.) (2007). Mechatronics for Safety, Security, Security and Dependability in a New Era (First, p. 461). Great Britain: Elsevier.

Gallego, Juan; Forner, A. (2008). Síntesis de la marcha robótica mediante la aproximación del ciclo límite: el robot bípedo esbirro. Science CSIC, 54-109.

Kuo, A. D. (2007). The six determinants of gait and the inverted pendulum analogy: A dynamic walking perspective. Human movement science, 26(4), 617-56.

Luo, G.; Houston, V.; Sasson, N.; Garbarini, M.; Beattie, A., \& Thongpop, C. (2011). Gait Characteristic Indices to Quantitatively Characterize Human Gait. Time, 23(3).

Nordin, M. (2004). Biomecánica Básica del Sistema Muscoesquelético-Nordin.pdf (Tercera Edción, p. 345). Madrid, España: McGraw Hill.

Perry, Jacquelin (1992). Gait Analysis: Normal and Pathological Function. Journal of Pediatric Orthopaedics (Vol. 12, p. 576). Slack Incorporated.

Perry, Jacquelin (1992). Normal Gait. Clin Orthop.

Pina, C. (2007). Humanoid Robots. (A. R. S. International, Ed.) (Primera Edición). Croatia: I-Tech.

Sandin, P. E. (2003). Robot Mechanics and Mechanical devices (Primera Edición, p. 299). New York: McGraw Hill.

Simon, S. R. (2004). Quantification of human motion: gait analysis-benefits and limitations to its application to clinical problems. Journal of biomechanics, 37(12), 1869-80.

V. Tikhanoff, P.; Fitzpatrick, F.; Nori, L.; Natale, G. M. and A. C. (2008). The iCub Humanoid Robot Simulator. Advanced Robotics, 1(1), 22-26.

Wang, L. (2003). Recent developments in human motion analysis. Pattern Recognition, 36(3), 585-601.

Zanchi, V. (2000). Quantitative human gait analysis. Simulation Practice and Theory, 8(1-2), 127-139.

Zivanovic (2006). Multi-Arm Cooperating Robots Microprocessor-Based And Intelligent Systems Engineering (p. 297). Netherlands: Advisor Board.

\section{(9) $(1) \Theta \Theta$}


